\title{
Adaptive Bias and Attitude Observer on the Special Orthogonal Group for True-North Gyrocompass Systems: Theory and Preliminary Results
}

\author{
Andrew R. Spielvogel \\ Johns Hopkings University \\ Baltimore, Maryland \\ Email: aspielv1@jhu.edu
}

\author{
Louis L. Whitcomb \\ Johns Hopkins University \\ Baltimore, Maryland \\ Email: 1lw@jhu.edu
}

\begin{abstract}
This paper reports an adaptive sensor bias estimator and attitude observer operating directly on $S O(3)$ for true-North gyrocompass systems that utilize six-degree of freedom inertial measurement units (IMUs) with three-axis accelerometers and three-axis gyroscopes (without magnetometers). Most presentday low-cost robotic vehicles employ attitude estimation systems that employ micro-electromechanical systems (MEMS) magnetometers, angular rate gyros, and accelerometers to estimate magnetic heading and attitude with limited heading accuracy. Present day MEMS gyros are not sensitive enough to dynamically detect Earth's rotation, and thus cannot be used to estimate trueNorth geodetic heading. In contrast, the reported gyrocompass system utilizes fiber optic gyroscope (FOG) IMU gyro and MEMS accelerometer measurements (without magnetometers) to dynamically estimate the instrument's time-varying attitude in real-time while the instrument is subject to a priori unknown rotations. Stability proofs, preliminary simulations, and a fullscale vehicle trial are reported that suggest the viability of the true-North gyrocompass system to provide dynamic real-time true-North heading, pitch, and roll while utilizing a comparatively low-cost FOG IMU.
\end{abstract}

\section{INTRODUCTION}

Accurate true-North heading and local level (roll and pitch) referenced to the local gravitational field (which we will refer to as true-North attitude) are critical components of highaccuracy navigation systems for a wide variety of robotic vehicles. The need for accurate true-North attitude estimation is particularly acute in the case of vehicles operating in global positioning system (GPS)-denied environments (such as underwater) and in magnetically compromised environments (such as near ferromagnetic structures, buildings, or natural local magnetic anomalies). Smaller and lower-cost vehicles represent an additional challenge due to their limited sensor budget, small physical size, and limited energy storage capacity.

Over the past decade the development of a new generation of small low-cost underwater vehicles (UVs) has begun to enable oceanographic, environmental assessment, and national security missions that were considered impractical or infeasible before (e.g. [3, 4, 25, 34, 38]). This new generation of UVs often employ low-cost navigation systems that presently limit them to missions requiring only low-precision navigation of
$\mathcal{O}(1-100) \mathrm{m}$ accuracy when submerged. High-end navigation approaches, of $\mathcal{O}(0.1-10) \mathrm{m}$ accuracy, traditionally require a Doppler sonar, costing $\$ 20 \mathrm{k}-\$ 50 \mathrm{k}$, and a North-seeking gyrocompass or inertial navigation system (INS), costing $\$ 50 \mathrm{k}$ $\$ 250 \mathrm{k}$. These high-end navigation approaches are largely incompatible with low-cost autonomous underwater vehicles (AUVs) with target total vehicle cost of $\$ 50 \mathrm{k}-\$ 250 \mathrm{k}$.

Moreover, the high cost, large size, and high powerconsumption of commercially available optical true-North seeking gyrocompasses is a principal barrier to the widespread use of high accuracy navigation for smaller and lower-cost UVs.

Small low-cost UVs typically employ micro-electromechanical systems (MEMS) inertial measurement units (IMUs) comprised of 3-axis MEMS magnetometers, gyros, and accelerometers to estimate local magnetic heading, pitch, and roll, typically to within several degrees of accuracy, but require careful soft-iron and hard-iron calibration and compensation to achieve these accuracies. Moreover, magnetic attitude sensors must be recalibrated for soft-iron and hard-iron errors whenever the vehicle physical configuration changes significantly (i.e. sensors or other payloads added or removed, etc.). Studies have shown that the accuracy of these magnetic heading sensors can be a principal error source in overall navigation solutions [15].

Recently, a new class of lower-cost ( $\sim$ 20k USD), compact and lower power fiber optic gyroscope (FOG) IMUs have become available - for example the commercial-off-theshelf (COTS) KVH 1775 FOG IMU (KVH Industries, Inc., Middletown, RI, USA) - that provide sensor accuracies sufficient for estimation of true-North heading, pitch and roll. This is in contrast to MEMS IMUs, which employ MEMS gyros that lack the sensitivity necessary to find true-North, and thus rely on MEMS magnetometers to sense magnetic heading.

True-North heading estimation differs from that of magnetic heading in that true-North heading uses the Earth's rotation to estimate the direction to the geodetic North Pole, while magnetic heading measures the Earth's magnetic field to estimate the direction to the magnetic North Pole. The gyroscope 
sensors (includes all MEMS IMUs) used in magnetic-North attitude sensors typically lack the sensitivity to detect the angular rate of Earth $\left(15^{\circ} / \mathrm{hr}\right)$ and are typically modeled as

$$
w_{m}(t)=w_{v}(t)+w_{b}+\eta(t)
$$

where $w_{m}(t)$ is the measured angular rate in instrument coordinates, $w_{v}(t)$ is the angular rate of the instrument with respect to the local North, East, down frame, $w_{b}$ is a constant measurement bias, and $\eta(t)$ is zero-mean Gaussian measurement noise. In contrast, true-North gyrocompass systems use high-end gyroscopes, such as three-axes FOGs, which are sensitive enough to measure Earth's angular rate and are typically modeled as

$$
w_{m}(t)=w_{E}(t)+w_{v}(t)+w_{b}+\eta(t)
$$

where $w_{m}(t), w_{v}(t), w_{b}$, and $\eta(t)$ are the same as in (1) and $w_{E}(t)$ is the angular rate of the Earth $\left(15^{\circ} / \mathrm{hr}\right)$. By fusing gyroscope and accelerometer measurements, true-North gyrocompass systems generate an estimate for the $w_{E}(t)$ component of the measured angular rate $w_{m}(t)$. Since the Earth's angular rate, $w_{E}(t)$, lies in the local North-down plane, the estimated angular-rate of Earth $\left(w_{E}(t)\right)$ and the estimated gravity vector can be fused to estimate the trueNorth direction. We define the local North-down plane to be plane that intersects the origin of the NED frame (defined in Section III-A) and spans the North and down directions.

This paper is the first report of a novel algorithm for estimating true-North attitude with real-time adaptive bias estimation of a dynamic (rotating) IMU without use of magnetometers. Preliminary simulation and experimental results of the reported true-North gyrocompass system employing a low-cost FOG IMU are reported.

This paper is organized as follows: Section II provides a literature review of attitude and sensor bias estimation. Section III gives an overview of preliminaries. Section IV reports the sensor bias and Earth-rate vector observer and stability proof. Section V presents the attitude observer and stability proof. Section VI presents preliminary numerical simulations and experimental results. Section VII summarizes and concludes.

\section{LITERATURE REVIEW}

\section{A. Attitude Estimation}

The majority of the attitude estimation literature addresses the case of magnetic heading attitude estimation using MEMS IMUs [7, 10, 11, 21, 22, 37]. Mahony et al. report an attitude nonlinear complementary filter on $S O(3)$ [19]. A recent study by Costanzi et al. explores utilizing a FOG for doing attitude estimation under unknown magnetic disturbances [5]. These studies however, differ from the current paper as they estimate magnetic North heading, while this paper presents an estimator for true-North heading.

Martinelli reports a method for estimating attitude using a three-axis accelerometer and three-axis gyroscope IMU and a monocular camera [20]. This approach however is impractical for many UV applications (e.g. when there is poor visibility, operating in the mid-water, operating in a region with a featureless bottom) and impossible for the many unmanned underwater vehicles (UUVs) with no cameras.

Previous studies by Spielvogel and Whitcomb suggest the practical utility of a low-cost FOG IMU as the primary sensor in a North-seeking gyrocompass system [31,32]. These studies assume that sensor biases have been calculated and compensated for a priori and rely on the differentiation of accelerometer measurements for estimating true-North.

In contrast to the previously reported observers by Spielvogel and Whitcomb, the present paper estimates true-North attitude without the need to differentiate accelerometer measurements and also addresses the problem of real time bias estimation for both gyros and accelerometers, which is essential for accurate true-north heading estimates.

\section{B. IMU Sensor Bias Estimation}

Several methods for IMU measurement bias estimation have been reported in recent years. Much of this literature, though, focuses on magnetometer bias estimation $[1,2,6,8,10,16$, $18,35]$.

Many papers report results for gyro sensor bias estimation. Most address MEMS gyro sensor bias estimation in which the angular rate due to Earth's rotation is ignored in the gyro measurement model. They use a measurement model similar to that of (1) and neglect the Earth rate term because it is dynamically undetectable with MEMS gyros.

George and Sukkarieh report an identifier for accelerometer and gyroscope sensor bias [9]. However, they utilize GPS which is unavailable to submerged vehicles.

Scandaroli et al. and Scandaroli and Morin [29, 30] also report a sensor bias estimator for 6-degrees of freedom (DOF) IMUs utilizing computer vision. This method though is dependent on the presence of a vision system, which requires identification markers and a camera system which is unavailable for many robotic vehicle (e.g. many underwater vehicles).

Metni et al. and Pflimlin et al. report nonlinear complementary filters for estimating attitude and gyroscope sensor bias [21, 22, 27]. While these estimators identify angular-rate sensor bias, they do not address linear acceleration sensor bias and do not distinguish the gyroscope sensor bias from Earth's angular velocity.

Spielvogel and Whitcomb address the problem of identifying and distinguishing the gyro bias from the Earth-rate signal [33]. However, their reported approach requires knowledge of the instrument's real-time attitude.

In contrast to previously reported estimators, the present paper proposes an algorithm for doing adaptive identification of both gyro and accelerometer sensor biases and true-North heading pitch, and roll on $S O(3)$ without a priori knowledge of the instrument's attitude.

\section{PREliminaries}

\section{A. Coordinate Frames}

We define the following coordinate frames: 
- Instrument Frame: A frame, denoted $(i)$, fixed in the IMU instrument.

- North-East-Down (NED) Frame: The North-East-Down (NED) frame, denoted $(N)$, has its $\mathrm{x}$-axis pointing North, its y-axis pointing East, its $\mathrm{z}$-axis pointing down, and its origin co-located with that of the instrument frame.

\section{B. Notation and Definitions}

For each vector, a leading superscript indicates the frame of reference and a following subscript indicates the signal source, thus ${ }^{N} w_{m}$ is the measured instrument angular velocity in the NED frame and ${ }^{i} a_{m}$ is the measured instrument linear acceleration in the instrument sensor frame.

For each rotation matrix a leading superscript and subscript indicates the frames of reference. For example, ${ }_{i}^{N} R$ is the rotation from the instrument frame to the NED frame.

Definition: $\mathcal{J}$ is defined as a function that maps a $3 \times 1$ vector to the corresponding $3 \times 3$ skew-symmetric matrix, $\mathcal{J}$ : $\mathbb{R}^{3} \rightarrow$ so(3). For $k \in \mathbb{R}^{3}$,

$$
\mathcal{J}(k)=\left[\begin{array}{ccc}
0 & -k_{3} & k_{2} \\
k_{3} & 0 & -k_{1} \\
-k_{2} & k_{1} & 0
\end{array}\right] .
$$

We define its inverse $\mathcal{J}^{-1}: s o(3) \rightarrow \mathbb{R}^{3}$, such that $\forall x \in \mathbb{R}^{3}$, $\mathcal{J}^{-1}(\mathcal{J}(x))=x$.

\section{Mathematical Background}

We will make use of the following mathematical facts:

Proposition: For $Q(t) \in s o(3)$, the rotation matrix $R(t)$ can be computed by Rodrigues' Equation [23]

$$
R(Q(t))=I_{3 \times 3}+\gamma(t) Q(t)+\kappa(t) Q(t)^{2}
$$

where

$$
\begin{aligned}
q(t) & =\mathcal{J}^{-1}(Q(t)) \\
\gamma(t) & =\frac{\sin (\|q(t)\|)}{\|q(t)\|} \\
\kappa(t) & =\frac{1-\cos (\|q(t)\|)}{\|q(t)\|^{2}} .
\end{aligned}
$$

Proposition: $\dot{q}(t)$ is related to $\dot{R}(t)$ by the mapping

$$
R^{T}(t) \dot{R}(t)=\mathcal{J}(A(q(t)) \dot{q}(t))
$$

where $A(q(t))$ is the right Jacobian of $R(q(t))=e^{\mathcal{J}(q(t))}$ with respect to the angular position vector $q(t) \in \mathbb{R}^{3} . A(q(t))$ and its inverse,

$$
A^{-1}(q(t))=I_{3 \times 3}+\alpha(t) \mathcal{J}(q(t))+\beta(t) \mathcal{J}(q(t))^{2}
$$

where

$$
\begin{aligned}
& \alpha(t)=-\frac{1}{2}, \\
& \beta(t)=\frac{1}{\|q(t)\|^{2}}-\frac{1+\cos (\|q(t)\|)}{2\|q(t)\| \sin (\|q(t)\|)},
\end{aligned}
$$

are reported by Park in [26].

If $A(q(t))$ is invertible, (8) can be rearranged as

$$
\dot{q}(t)=A^{-1}(q(t)) \mathcal{J}^{-1}\left(R^{T}(t) \dot{R}(t)\right) .
$$

\section{Sensor Model}

The sensor measurement models for angular rate and linear acceleration are

$$
\begin{aligned}
{ }^{i} w_{m}(t) & ={ }^{i} w_{E}(t)+{ }^{i} w_{v}(t)+{ }^{i} w_{b}+{ }^{i} \eta_{w}(t) \\
{ }^{i} a_{m}(t) & ={ }^{i} a_{g}(t)+{ }^{i} a_{v}(t)+{ }^{i} a_{b}+{ }^{i} \eta_{a}(t) \\
{ }^{i} w_{e}(t) & ={ }^{i} w_{E}(t)+{ }^{i} w_{v}(t)+{ }^{i} w_{b} \\
{ }^{i} a_{e}(t) & ={ }^{i} a_{g}(t)+{ }^{i} a_{v}(t)+{ }^{i} a_{b}
\end{aligned}
$$

where ${ }^{i} w_{m}(t)$ is the IMU measured angular-rate, ${ }^{i} w_{e}(t)$ is the true noise-free angular-rate, ${ }^{i} w_{E}(t)$ is the true angular velocity due to the rotation of the Earth, ${ }^{i} w_{v}(t)$ is the true angular velocity due to the rotation of the instrument with respect to the NED frame, ${ }^{i} w_{b}$ is the angular velocity sensor bias offset, ${ }^{i} \eta_{w}(t)$ is the zero-mean Gaussian angular velocity sensor noise, ${ }^{i} a_{m}(t)$ is the IMU measured linear acceleration, ${ }^{i} a_{e}(t)$ is the true noise-free linear acceleration, ${ }^{i} a_{g}(t)$ is the true linear acceleration due to gravity and the Earth's rotation, ${ }^{i} a_{v}(t)$ is the instrument's true linear acceleration with respect to Earth, ${ }^{i} a_{b}$ is the linear accelerometer sensor bias, and ${ }^{i} \eta_{a}(t)$ is the zero-mean Gaussian linear accelerometer sensor noise.

For many robotic vehicles, the gravitational field ${ }^{i} a_{g}(t)$ dominates the vehicle linear acceleration $\left({ }^{i} a_{v}(t)\right)$. Thus, it is common to use

$$
{ }^{i} a_{e}(t) \approx{ }^{i} a_{g}(t)+{ }^{i} a_{b}
$$

as a low-frequency estimate of (16) ([5, 19, 27, 37]). Given (17), the sensor measurement model becomes

$$
\begin{aligned}
{ }^{i} w_{m}(t) & ={ }^{i} w_{E}(t)+{ }^{i} w_{v}(t)+{ }^{i} w_{b}+{ }^{i} \eta_{w}(t) \\
{ }^{i} a_{m}(t) & ={ }^{i} a_{g}(t)+{ }^{i} a_{b}+{ }^{i} \eta_{a}(t) \\
{ }^{i} w_{e}(t) & ={ }^{i} w_{E}(t)+{ }^{i} w_{v}(t)+{ }^{i} w_{b} \\
{ }^{i} a_{e}(t) & ={ }^{i} a_{g}(t)+{ }^{i} a_{b} .
\end{aligned}
$$

Section VI-G shows the proposed algorithms perform well in the experimental trial, thus justifying the neglection of the vehicle acceleration term ${ }^{i} a_{v}(t)$ in (17).

\section{E. Earth's Angular Velocity}

Since we know that ${ }^{i} w_{E}(t)$ lies in the North-down plane, we can write the signal ${ }^{i} w_{E}(t)$ as the sum of its North and down components as

$$
{ }^{i} w_{E}(t)={ }^{i} w_{E_{n}}(t)+{ }^{i} w_{E_{d}}(t) .
$$

In addition, we know that ${ }^{i} a_{g}(t)$ is in the direction of up and what the values of the magnitudes (function of latitude) of the two components of ${ }^{i} w_{E}(t)$ are. We can substitute into (22) resulting in

$$
{ }^{i} w_{E}={ }^{i} w_{E_{n}}(t)+\gamma^{i} a_{g}(t)
$$

where

$$
\gamma=\frac{\left\|^{N} w_{E_{d}}\right\|}{\left\|{ }^{N} a_{g}\right\|}
$$




\section{Sensor Bias And $w_{E_{n}}$ ObServer}

This section reports the derivation and stability analysis of an adaptive sensor bias and Earth-rate vector (the North component) observer for six-DOF IMUs equipped with a three-axis accelerometer and three-axis angular rate gyroscope.

Note that the gyroscope must be sensitive enough to detect Earth-rate. The measurement noise of present-day angular rate gyros in MEMS IMUs is orders of magnitude larger than is needed to detect the extremely minute signal of the Earth's rotation rate $\left(15^{\circ} / \mathrm{hr}\right)$, thus, MEMS IMUs cannot be utilized to dynamically estimate directly true-North heading. At present, true-North attitude can only be successfully instrumented with high-end, angular-rate gyros that employ ring laser gyro (RLG) or FOG angular rate sensors, or that employ large inertialgrade mechanical gyrocompasses. The present paper reports a system that successfully estimates true-North attitude utilizing a new class of lower-cost FOG IMUs.

\section{A. System Model}

We consider the system model

$$
\begin{aligned}
{ }^{N} a_{g} & ={ }_{i}^{N} R(t){ }^{i} a_{g}(t) \\
& ={ }_{i}^{N} R(t)\left({ }^{i} a_{e}(t)-{ }^{i} a_{b}\right) \\
{ }^{N} w_{E_{n}} & ={ }_{i}^{N} R(t){ }^{i} w_{E_{n}}(t) .
\end{aligned}
$$

Differentiating (25) and (26) and rearranging terms yields

$$
\begin{aligned}
{ }^{i} \dot{a}_{e}(t) & =-\mathcal{J}\left({ }^{i} w_{e}(t)-{ }^{i} w_{b}-{ }^{i} w_{E}(t)\right){ }^{i} a_{g}(t) \\
{ }^{i} \dot{w}_{E_{n}}(t) & =-\mathcal{J}\left({ }^{i} w_{e}(t)-{ }^{i} w_{b}-{ }^{i} w_{E}(t)\right){ }^{i} w_{E_{n}}(t) .
\end{aligned}
$$

Substituting (23) into (27) and (28) results in

$$
\begin{aligned}
{ }^{i} \dot{a}_{e}(t)= & -\mathcal{J}\left({ }^{i} w_{e}(t)-{ }^{i} w_{b}-{ }^{i} w_{E_{n}}(t)-\gamma^{i} a_{g}(t)\right){ }^{i} a_{g}(t) \\
= & -\mathcal{J}\left({ }^{i} w_{e}(t)-{ }^{i} w_{b}-{ }^{i} w_{E_{n}}(t)\right){ }^{i} a_{g}(t) \\
\approx & -\mathcal{J}\left({ }^{i} w_{e}(t)-{ }^{i} w_{b}-{ }^{i} w_{E_{n}}(t)\right){ }^{i} a_{e}(t) \\
& +\mathcal{J}\left({ }^{i} w_{e}(t)\right){ }^{i} a_{b} \\
{ }^{i} \dot{w}_{E_{n}}= & -\mathcal{J}\left({ }^{i} w_{e}(t)-{ }^{i} w_{b}-{ }^{i} w_{E_{n}}(t)-\gamma^{i} a_{g}(t)\right){ }^{i} w_{E_{n}}(t) \\
= & -\mathcal{J}\left({ }^{i} w_{e}(t)-{ }^{i} w_{b}-\gamma^{i} a_{g}(t)\right){ }^{i} w_{E_{n}}(t) \\
\approx & -\mathcal{J}\left({ }^{i} w_{e}(t)-\gamma^{i} a_{e}(t)\right){ }^{i} w_{E_{n}}(t)
\end{aligned}
$$

where we are making the approximations that $\mathcal{J}\left({ }^{i} w_{b}+{ }^{i} w_{E_{n}}(t)\right){ }^{i} a_{b} \approx 0$ and $\mathcal{J}\left({ }^{i} w_{b}-\gamma^{i} a_{b}\right){ }^{i} w_{E_{n}} \approx 0$, since the cross products between sensor biases and the Earth rate vector are orders of magnitude smaller than the other signals.

\section{B. Sensor Bias and $w_{E_{n}}$ Observer}

We consider the observer system model

$$
\begin{aligned}
{ }^{i} \dot{\hat{a}}_{e}(t)= & -\mathcal{J}\left({ }^{i} w_{e}(t)-{ }^{i} \hat{w}_{b}(t)-{ }^{i} \hat{w}_{E_{n}}(t)\right){ }^{i} \hat{a}_{e}(t) \\
& +\mathcal{J}\left({ }^{i} w_{e}(t)\right){ }^{i} \hat{a}_{b}(t)-k_{a}(t) \Delta a(t) \\
{ }^{i} \dot{\hat{w}}_{E_{n}}(t)= & -\mathcal{J}\left({ }^{i} w_{e}(t)-\gamma^{i} a_{e}(t)\right){ }^{i} \hat{w}_{E_{n}}(t) \\
& -k_{E}(t) \mathcal{J}\left({ }^{i} a_{e}(t)\right) \Delta a(t) \\
{ }^{i} \dot{\hat{w}}_{b}(t)= & -k_{b_{w}}(t) \mathcal{J}\left({ }^{i} a_{e}(t)\right) \Delta a(t) \\
{ }^{i} \dot{\hat{a}}_{b}(t)= & k_{b_{a}}(t) \mathcal{J}\left({ }^{i} w_{e}(t)\right) \Delta a(t)
\end{aligned}
$$

where $k_{a}(t), k_{E}(t), k_{b_{w}}(t)$, and $k_{b_{a}}(t)$ are positive nonincreasing scalar gains, ${ }^{i} \hat{a}_{e}(t),{ }^{i} \hat{w}_{E_{n}}(t),{ }^{i} \hat{w}_{b}(t)$, and ${ }^{i} \hat{a}_{b}(t)$ are the estimates of ${ }^{i} a_{e}(t),{ }^{i} w_{E_{n}}(t),{ }^{i} w_{b}$, and ${ }^{i} a_{b}$ respectively, and

$$
\begin{aligned}
\Delta a(t) & ={ }^{i} \hat{a}_{e}(t)-{ }^{i} a_{e}(t) \\
\Delta w_{E_{n}}(t) & ={ }^{i} \hat{w}_{E_{n}}(t)-{ }^{i} w_{E_{n}}(t) \\
\Delta w_{b}(t) & ={ }^{i} \hat{w}_{b}(t)-{ }^{i} w_{b} \\
\Delta a_{b}(t) & ={ }^{i} \hat{a}_{b}(t)-{ }^{i} a_{b}
\end{aligned}
$$

are the error terms.

C. Error System

The resulting error system is

$$
\begin{aligned}
\Delta \dot{a}(t)= & -\mathcal{J}\left({ }^{i} a_{e}(t)\right) \Delta w_{b}(t)-\mathcal{J}\left({ }^{i} a_{e}(t)\right) \Delta w_{E_{n}}(t) \\
& +\mathcal{J}\left({ }^{i} w_{e}(t)\right) \Delta a_{b}(t)-k_{a}(t) \Delta a(t) \\
& -\mathcal{J}\left({ }^{i} w_{e}(t)-{ }^{i} \hat{w}_{b}(t)-{ }^{i} \hat{w}_{E_{n}}(t)\right) \Delta a(t) \\
\Delta \dot{w}_{E_{n}}(t)= & -\mathcal{J}\left({ }^{i} w_{e}(t)-\gamma^{i} a_{e}(t)\right) \Delta w_{E_{n}}(t) \\
& -k_{E}(t) \mathcal{J}\left({ }^{i} a_{e}(t)\right) \Delta a(t) \\
\Delta \dot{w}_{b}(t)= & -k_{b_{w}}(t) \mathcal{J}\left({ }^{i} a_{e}(t)\right) \Delta a(t) \\
\Delta \dot{a}_{b}(t)= & k_{b_{a}}(t) \mathcal{J}\left({ }^{i} w_{e}(t)\right) \Delta a(t) .
\end{aligned}
$$

\section{Stability}

Consider the Lyapunov function candidate

$$
\begin{aligned}
V= & \frac{1}{2} \Delta a^{T}(t) \Delta a(t)+\frac{1}{2 k_{E}(t)} \Delta w_{E_{n}}^{T}(t) \Delta w_{E_{n}}(t) \\
& +\frac{1}{2 k_{b_{w}}(t)} \Delta w_{b}^{T}(t) \Delta w_{b}(t)+\frac{1}{2 k_{b_{a}}(t)} \Delta a_{b}^{T}(t) \Delta a_{b}(t) .
\end{aligned}
$$

Differentiating (43) results in

$$
\begin{aligned}
\dot{V}= & \left(-\Delta a^{T}(t) \mathcal{J}\left({ }^{i} a_{e}(t)\right)+\Delta a^{T}(t) \mathcal{J}\left({ }^{i} a_{e}(t)\right)\right) \Delta w_{b}(t) \\
& +\left(-\Delta a^{T}(t) \mathcal{J}\left({ }^{i} a_{e}(t)\right)+\Delta a^{T}(t) \mathcal{J}\left({ }^{i} a_{e}(t)\right)\right. \\
& \left.+\frac{1}{k_{E}} \Delta w_{E_{n}}^{T}(t) \mathcal{J}\left({ }^{i} w_{e}(t)-\gamma^{i} a_{e}(t)\right)\right) \Delta w_{E_{n}}(t) \\
& +\left(\Delta a^{T}(t) \mathcal{J}\left({ }^{i} w_{e}(t)\right)-\Delta a^{T}(t) \mathcal{J}\left({ }^{i} w_{e}(t)\right)\right) \Delta a_{b}(t) \\
& -\Delta a^{T}(t) \mathcal{J}\left({ }^{i} w_{e}(t)-{ }^{i} \hat{w}_{b}(t)-{ }^{i} \hat{w}_{E_{n}}(t)\right) \Delta a(t) \\
& -k_{a}\|\Delta a(t)\|^{2}+\frac{1}{2 \dot{k}_{E}(t)}\left\|\Delta w_{E_{n}}(t)\right\|^{2} \\
& +\frac{1}{2 \dot{k}_{b_{w}}(t)}\left\|\Delta w_{b}(t)\right\|^{2}+\frac{1}{2 \dot{k}_{b_{a}}(t)}\left\|\Delta a_{b}(t)\right\|^{2} \\
= & -k_{a}\|\Delta a(t)\|^{2}+\frac{1}{2 \dot{k}_{E}(t)}\left\|\Delta w_{E_{n}}(t)\right\|^{2} \\
& +\frac{1}{2 \dot{k}_{b_{w}}(t)}\left\|\Delta w_{b}(t)\right\|^{2}+\frac{1}{2 \dot{k}_{b_{a}}(t)}\left\|\Delta a_{b}(t)\right\|^{2} .
\end{aligned}
$$

Since $k_{E}(t), k_{b_{w}}(t)$, and $k_{b_{a}}(t)$ are non-increasing scalars, the time derivative of the Lyapunov function is negative semidefinite and the observer is locally stable. Additional arguments beyond the scope of this paper are required to show local asymptotic stability; however preliminary numerical simulations and experimental results demonstrate the estimator to converge. 


\section{Attitude ObServer}

This section derives an attitude observer that updates directly on $S O(3)$ for doing true-North attitude estimation. The observer is inspired by the reserch of Mahony et al. on Nonlinear Complementary Filters on $S O(3)$ [19] and research by Kinsey and Whitcomb on adaptive identification on $S O(3)$ [14].

We choose the update law

$$
\begin{aligned}
{ }_{i}^{N} \dot{\hat{R}}(t)= & { }_{i}^{N} \hat{R}(t) \mathcal{J}\left(\tilde{a}(t)+\tilde{n}(t)+{ }^{i} w_{e}(t)-{ }^{i} w_{b}\right. \\
& \left.-{ }_{i}^{N} \hat{R}^{T}(t)^{N} w_{E}\right) .
\end{aligned}
$$

where the local-level $\tilde{a}(t)$ and heading $\tilde{n}(t)$ error terms are defined, respectively, as

$$
\begin{aligned}
& \tilde{a}(t)=k_{g}(t) \mathcal{J}\left({ }^{i} a_{g}(t)\right){ }_{i}^{N} \hat{R}^{T}(t)^{N} a_{g} \\
& \tilde{n}(t)=k_{n}(t) P \mathcal{J}\left({ }^{i} w_{E_{n}}(t)\right){ }_{i}^{N} \hat{R}^{T}(t)^{N} w_{E_{n}}
\end{aligned}
$$

where

$$
\begin{aligned}
& P={ }_{i}^{N} \hat{R}^{T}(t)^{N} \bar{a}_{g}{ }^{N} \bar{a}_{g i}^{T N} \hat{R}(t), \\
& { }^{N} a_{g}=-\left(I_{3 \times 3}+\frac{1}{g_{0}} \mathcal{J}\left({ }^{N} w_{E}\right)^{2}\right) \mathbf{e}_{3}, \\
& { }^{N} \bar{a}_{g}=\frac{{ }^{N} a_{g}}{\left\|{ }^{N} a_{g}\right\|}, \\
& { }^{N} w_{E_{n}}=\left(I_{3 \times 3}-\mathbf{e}_{3} \mathbf{e}_{3}^{T}\right)^{N} w_{E}, \\
& \mathbf{e}_{3}=\left[\begin{array}{lll}
0 & 0 & 1
\end{array}\right]^{T},
\end{aligned}
$$

$g_{0}$ is the magnitude of gravity $\left(\sim 9.81 \mathrm{~m} / \mathrm{s}^{2}\right)$, and $k_{g}(t)>0$ and $k_{n}(t)>0$ are scalar gains.

The parameter error is defined as

$$
\tilde{R}(t)={ }_{i}^{N} R^{T}(t)_{i}^{N} \hat{R}(t)
$$

with the time derivative

$$
\begin{aligned}
\dot{\tilde{R}}(t)= & { }_{i}^{N} \dot{R}^{T}(t)_{i}^{N} \hat{R}(t)+{ }_{i}^{N} R^{T}(t){ }_{i}^{N} \hat{\hat{R}}(t) \\
= & -\mathcal{J}\left({ }^{i} w_{v}(t)\right) \tilde{R}(t) \\
& +\tilde{R}(t) \mathcal{J}\left(\tilde{a}(t)+\tilde{n}(t)+{ }^{i} w_{e}(t)-{ }^{i} w_{b}\right. \\
& \left.-{ }_{i}^{N} \hat{R}^{T}(t)^{N} w_{E}\right) \\
= & \tilde{R}(t) \mathcal{J}\left(\tilde{a}(t)+\tilde{n}(t)+{ }^{i} w_{e}(t)-{ }^{i} w_{b}\right. \\
& \left.-\tilde{R}^{T}(t)^{i} w_{E}(t)-\tilde{R}^{T}(t)^{i} w_{v}(t)\right) \\
= & \tilde{R}(t) \mathcal{J}\left(\tilde{a}(t)+\tilde{n}(t)+{ }^{i} w_{E v}(t)-\tilde{R}^{T}(t)^{i} w_{E v}(t)\right)
\end{aligned}
$$

where

$$
{ }^{i} w_{E v}(t)={ }^{i} w_{E}(t)+{ }^{i} w_{v}(t) .
$$

\section{A. Stability}

The Lyapunov candidate function is

$$
V(\tilde{R}(t))=\frac{1}{2}\|\tilde{q}(t)\|^{2} .
$$

The time derivative of the Lyapunov candidate function is

$$
\dot{V}(\tilde{R}(t))=\tilde{q}^{T}(t) \dot{\tilde{q}}(t) .
$$

Substituting (12) into (57) results in

$$
\dot{V}(\tilde{R}(t))=\tilde{q}^{T}(t)\left(A^{-1}(\tilde{q}(t)) \mathcal{J}^{-1}\left(\tilde{R}^{T}(t) \dot{\tilde{R}}(t)\right)\right)
$$

and substituting in (9) and (54) results is

$$
\dot{V}(\tilde{R}(t))=\tilde{q}^{T}(t)\left(\tilde{a}(t)+\tilde{n}(t)+{ }^{i} w_{E v}(t)-\tilde{R}^{T}(t)^{i} w_{e}(t)\right) .
$$

Substituting (46), (47), and (4) into (59) results in

$$
\begin{aligned}
\dot{V}(\tilde{R}(t))= & \tilde{q}^{T}(t)(\tilde{a}(t)+\tilde{n}(t)) \\
= & k_{g}(t) \tilde{q}^{T}(t) \mathcal{J}\left({ }^{i} a_{g}(t)\right){ }_{i}^{N} \hat{R}^{T}(t)^{N} a_{g} \\
& +k_{n}(t) \tilde{q}^{T}(t) P \mathcal{J}\left({ }^{i} w_{E_{n}}(t)\right){ }_{i}^{N} \hat{R}^{T}(t)^{N} w_{E_{n}} \\
= & k_{g}(t) \tilde{q}^{T}(t) \mathcal{J}\left({ }^{i} a_{g}(t)\right) \tilde{R}^{T}(t)^{i} a_{g}(t) \\
& +k_{n}(t) \tilde{q}^{T}(t) P \mathcal{J}\left({ }^{i} w_{E_{n}}(t)\right) \tilde{R}^{T}(t)^{i} w_{E_{n}} \\
= & -k_{g}(t) \tilde{\gamma}(t) \tilde{q}^{T}(t) \mathcal{J}\left({ }^{i} a_{g}(t)\right) \mathcal{J}(\tilde{q}(t))^{i} a_{g}(t) \\
& -k_{n}(t) \tilde{\gamma}(t) \tilde{q}^{T}(t) P \mathcal{J}\left({ }^{i} w_{E_{n}}(t)\right) \mathcal{J}(\tilde{q}(t))^{i} w_{E_{n}} .
\end{aligned}
$$

Using the fact that $P^{2}=P$ for projection matrices and that orthogonal matrices distribute across the cross product, the time derivative simplifies to

$$
\begin{aligned}
\dot{V}(\tilde{R}(t))= & -k_{g}(t) \tilde{\gamma}(t)\left\|\mathcal{J}\left({ }^{i} a_{g}(t)\right) \tilde{q}(t)\right\|^{2} \\
& -k_{n}(t) \tilde{\gamma}(t) \tilde{q}^{T}(t) P \mathcal{J}\left(P^{i} w_{E_{n}}(t)\right) \mathcal{J}(P \tilde{q}(t)) P^{i} w_{E_{n}} \\
= & -k_{g}(t) \tilde{\gamma}(t)\left\|\mathcal{J}\left({ }^{i} a_{g}(t)\right) \tilde{q}(t)\right\|^{2} \\
& -k_{n}(t) \tilde{\gamma}(t)\left\|P \mathcal{J}\left({ }^{i} w_{E_{n}}(t)\right) \tilde{q}(t)\right\|^{2} \\
\leq & 0 .
\end{aligned}
$$

Thus, the time derivative of the Lyapunov function is negative semidefinite and the observer is locally stable. Additional arguments beyond the scope of this paper are required to show local asymptotic stability; however preliminary numerical simulations and experimental results demonstrate the estimator to asymptotically converge to the true attitude.

\section{Gyrocompass System Preliminary Evaluation}

The estimates of, ${ }^{i} w_{E_{n}},{ }^{i} w_{b}$, and ${ }^{i} a_{b}$ from the bias observer presented in Section IV are utilized in real-time by the attitude observer of Section V as follows:

$$
\begin{aligned}
{ }^{i} w_{E_{n}}(t) & ={ }^{i} \hat{w}_{E_{n}}(t), \\
{ }^{i} a_{g}(t) & ={ }^{i} a_{e}(t)-{ }^{i} \hat{a}_{b}(t), \\
{ }^{i} w_{b} & ={ }^{i} \hat{w}_{b}(t) .
\end{aligned}
$$

The combined use of the reported bias estimator (for accelerometers and angular rate sensor bias calibration on-thefly) and the reported true-North attitude estimator will be termed the "gyrocompass system" in the following sections.

The gyrocompass system is preliminarily evaluated in three numerical simulations and one UV trial. 


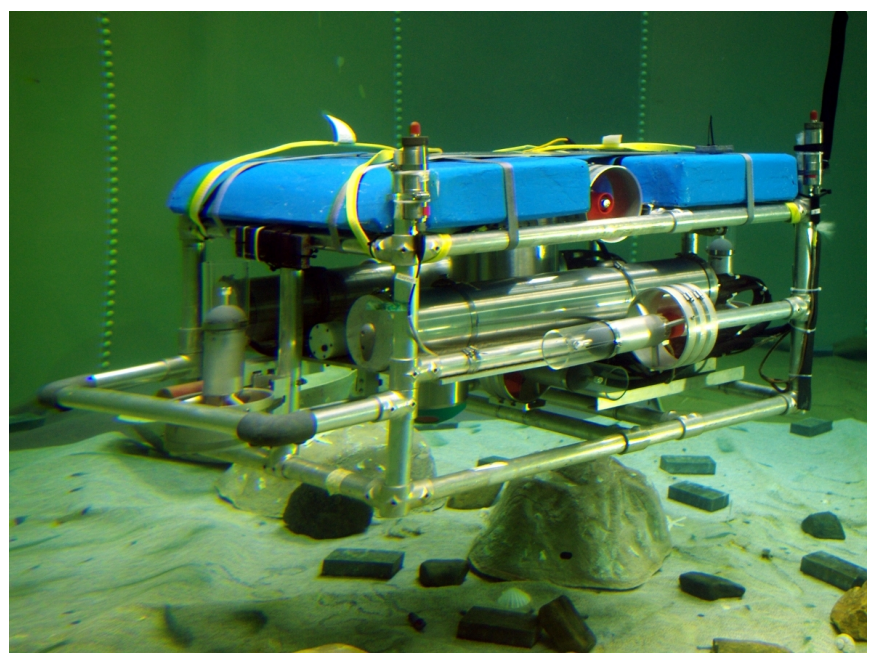

Fig. 1: Fully actuated ROV used for vehicle trial.

Note that in the derivation and stability proofs presented in Sections IV and V, the noise free case (20-21) of the measurement model is used. In the evaluation of the gyrocompass system, the noisy measurement model (18-19) is used instead.

\section{A. Test Facility}

An experimental trial was performed with a remotely operated vehicle (ROV) equipped with a KVH 1775 FOG IMU (KVH Industries, Inc., Middletown, RI, USA) in the facility's $7.5 \mathrm{~m}$ diameter $\times 4 \mathrm{~m}$ deep fresh water tank. The ROV is a fully actuated (six-DOF) vehicle with six $1.5 \mathrm{~kW}$ DC brushless electric thrusters and employs a suite of sensors commonly employed on deep submergence underwater vehicles. This includes a high-end INS, the iXBlue PHINS III (iXBlue SAS, Cedex, France) [12, 13], that is used as a "ground-truth" comparison during the experimental trial. The PHINS is a high-end INS $(\sim \$ 120 \mathrm{k})$ with roll, pitch, heading accuracies of $0.01^{\circ}, 0.01^{\circ}, 0.05^{\circ} / \cos$ (latitude), respectively [12]. Figure 1 shows the ROV operating in the test tank.

\section{B. Modified Linear Acceleration Bias Update Law}

In our initial experiments we observed that, over time, the magnitude of the estimated gravity vector would gradually evolve to a physically unrealiztic value. Using the additional information that we know the magnitude of ${ }^{i} a_{g}(t)$ at a given latitude and longitude, the observer system model, (34), for the linear acceleration bias was modified to preserve the correct a priori known magnitude of the acceleration estimation as it is adapted as follows:

$$
\begin{aligned}
{ }^{i} \dot{\hat{a}}_{b}(t)= & k_{b_{a}}(t) \mathcal{J}\left({ }^{i} w_{e}(t)\right) \Delta a(t) \\
& +k_{f} \frac{{ }^{i} \hat{a}_{g}^{T}(t)^{i} w_{e}(t)}{\left|{ }^{i} \hat{a}_{g}^{T}(t)^{i} w_{e}(t)\right|} \frac{{ }^{i} w_{e}(t)}{\left\|{ }^{i} w_{e}(t)\right\|}\left(\left\|{ }^{i} \hat{a}_{g}(t)\right\|-\left\|^{N} a_{g}(t)\right\|\right)
\end{aligned}
$$

where

$$
{ }^{i} \hat{a}_{g}(t)={ }^{i} \hat{a}_{e}(t)-{ }^{i} \hat{a}_{b}(t)
$$

and $k_{f}>0$. We have made this modification so that the linear acceleration bias also evolves along ${ }^{i} w_{e}(t)$ instead of only in the image of $\mathcal{J}\left({ }^{i} w_{e}(t)\right)$. The resulting entire observer system model from Section IV becomes

$$
\begin{aligned}
i \dot{\hat{a}}_{e}(t)= & -\mathcal{J}\left({ }^{i} w_{e}(t)-{ }^{i} \hat{w}_{b}(t)-{ }^{i} \hat{w}_{E_{n}}(t)\right){ }^{i} \hat{a}_{e}(t) \\
& +\mathcal{J}\left({ }^{i} w_{e}(t)\right){ }^{i} \hat{a}_{b}(t)-k_{a}(t) \Delta a(t) \\
{ }^{i} \dot{\hat{w}}_{E_{n}}(t)= & -\mathcal{J}\left({ }^{i} w_{e}(t)-\gamma^{i} a_{e}(t)\right){ }^{i} \hat{w}_{E_{n}}(t) \\
& -k_{E}(t) \mathcal{J}\left({ }^{i} a_{e}(t)\right) \Delta a(t) \\
{ }^{i} \dot{\hat{w}}_{b}(t)= & -k_{b_{w}}(t) \mathcal{J}\left({ }^{i} a_{e}(t)\right) \Delta a(t) \\
{ }^{i} \dot{\hat{a}}_{b}(t)= & k_{b_{a}}(t) \mathcal{J}\left({ }^{i} w_{e}(t)\right) \Delta a(t) \\
& +k_{f} \frac{{ }^{i} \hat{a}_{g}^{T}(t)^{i} w_{e}(t)}{\left|{ }^{i} \hat{a}_{g}^{T}(t)^{i} w_{e}(t)\right|} \frac{{ }^{i} w_{e}(t)}{\left\|{ }^{i} w_{e}(t)\right\|}\left(\left\|^{i} \hat{a}_{g}(t)\right\|-\left\|^{N} a_{g}(t)\right\|\right)
\end{aligned}
$$

This modified observer has performed well in preliminary simulation studies and experimental evaluation. We are presently working to develop a concise stability analysis for this variant of the algorithm, and hope to report it in future publications.

\section{Gain Selection}

Gain selection is important to the performance of the gyrocompass system. As with most adaptive systems that rely on persistence of excitation $[24,28]$ to converge to the true parameter values, the rate of convergence is dependent on the amount of excitation the system is experiencing and gains. In order for consistent rates of convergence across different instrument rotation rates, we have found it to be helpful for the gains in the observer to be adaptive with respect to the system's excitation. The gains used in the simulations and experiment were empirically selected as follows:

$$
\begin{aligned}
k_{g} & =0.1 \\
\dot{k}_{n}(t) & =\left\{\begin{array}{cc}
-0.0005, & k_{n}>0.0025 \\
0, & k_{n} \leq 0.0025
\end{array}\right. \\
k_{n}(0) & =1 \\
k_{a} & =0.01 \\
k_{E} & =0.000001 \\
\dot{k}_{b_{w}}(t) & =\left\{\begin{array}{c}
-0.000000003 * \|^{i} w_{e}(t) \\
k_{b_{w}}(0)
\end{array}=0.000001\right. \\
k_{b_{a}} & =1 \\
k_{f} & =0.0025 .
\end{aligned}
$$$$
\dot{k}_{b_{w}}(t)=\left\{\begin{array}{cl}
-0.000000003 *\left\|^{i} w_{e}(t)\right\|, & k_{b_{w}}>0.0000005 \\
0, & k_{b_{w}} \leq 0.0000005
\end{array}\right.
$$

The purpose of these time varying gains is to begin with large gains while the system is initializing, and to reduce the gains as the system errors converge in response to the vehicle angular motion. These time varying gains are included in the stability analysis resported in Section IV.

\section{Simulation Setup}

The gyrocompass system is evaluated in three numerical simulations. 

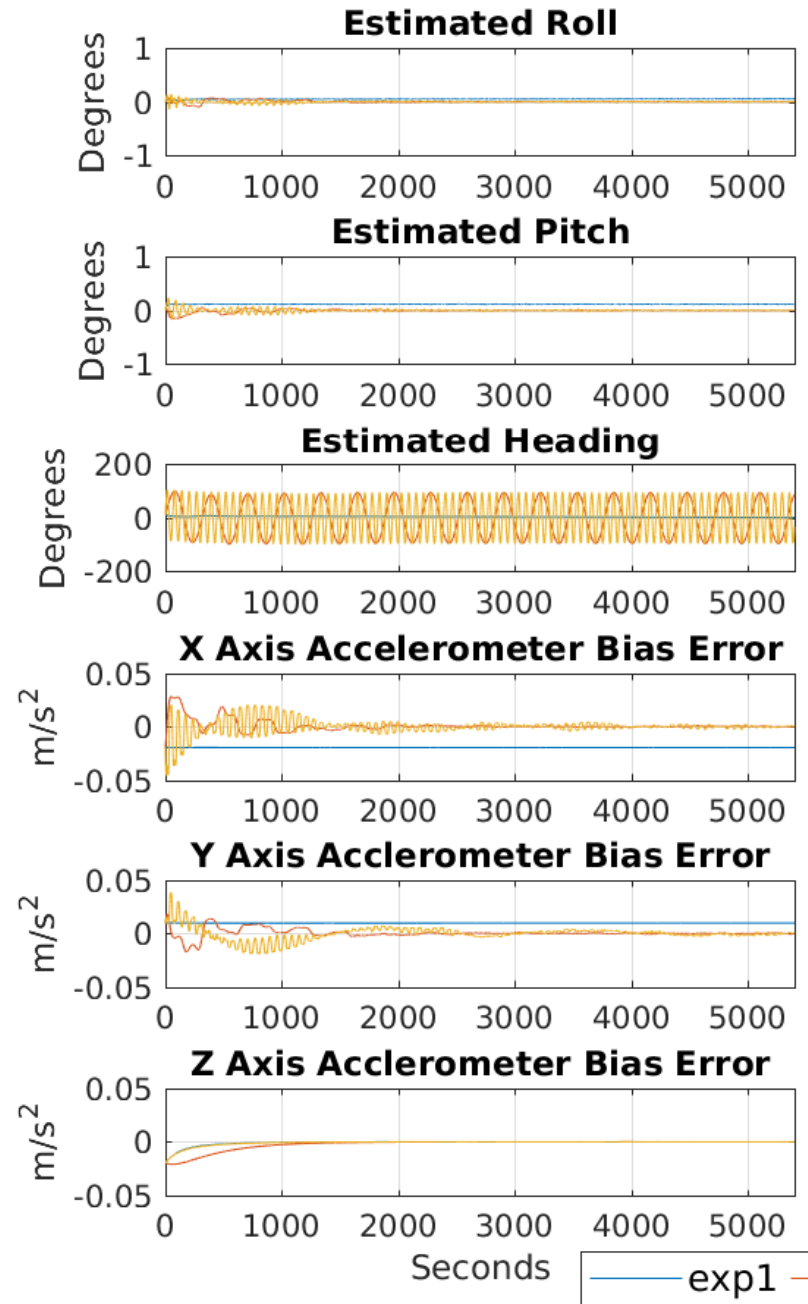
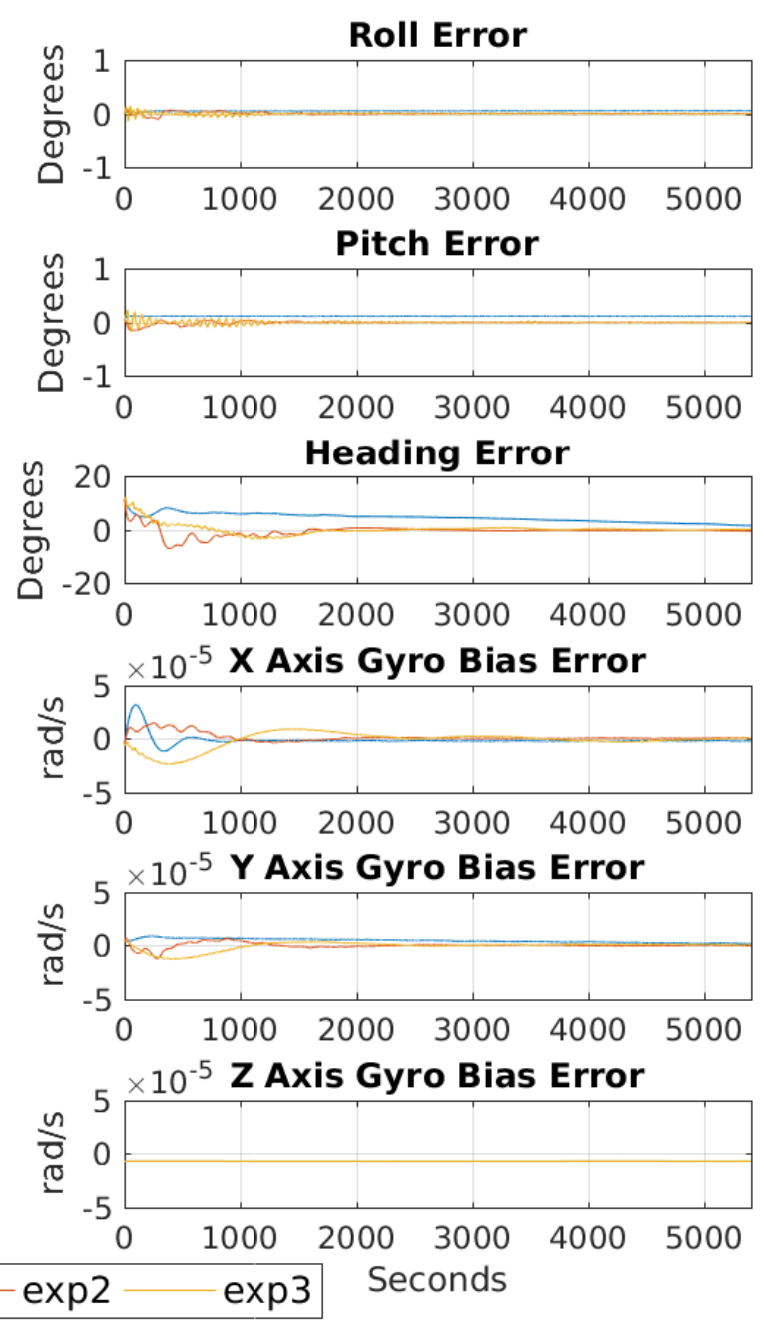

Fig. 2: Results of three numerical simulations.

- Sensor measurement sampling was simulated at $1 \mathrm{kHz}$.

- Simulations include sensor biases consistent in magnitude to those seen in KVH 1775 IMUs.

- Simulations were for a latitude of $39.32^{\circ} \mathrm{N}$.

- Simulations included sensor measurements with sensor noise representative of the KVH 1775 FOG IMU (used ${ }^{i} a_{m}(t)$ and ${ }^{i} w_{m}(t)$ instead of ${ }^{i} a_{e}(t)$ and $\left.{ }^{i} w_{e}(t)\right)$. Angular velocity sensor and linear accelerometer sensor noises are computed from the IMU's specifications [17], as per [36], and confirmed by the authors experimentally to be $\sigma_{w}=$ $6.32 \times 10^{-3} \mathrm{rad} / \mathrm{s}$ and $\sigma_{a}=0.0037 \mathrm{~g}$.

- The exp1 simulation experienced no rotation.

- The exp2 and exp3 simulations experienced changes in heading.

\section{E. Simulation Results}

The estimated attitude and sensor bias errors for the three simulations are shown in Figure 2. The exp1 simulation results show that when the instrument is not excited via rotations, the sensor bias do not converge to their true values. This is consistent with adaptive identifiers which rely on persistence of excitation ([24, 28]). In the exp2 and exp3 simulations, the attitude converges. Specifically, the simulations show (after the system has converged) the gyrocompass system to estimate roll and pitch within $0.25^{\circ}$ and heading within $0.5^{\circ}$.

\section{F. Experimental Setup}

The gyrocompass system is evaluated with a preliminary vehicle trial employing a comparatively low-cost $(\sim \$ 20 \mathrm{k}$ USD) FOG KVH 1775 IMU (KVH Industries, Inc., Middletown, RI, USA).

- The KVH 1775 FOG IMU was sampled at 5kHz.

- The KVH 1775 FOG IMU was aligned via a fixture to the ROV's iXSEA PHINS INS (iXblue SAS, Cedex, France). The PHINS is used as ground truth during our experimental evaluation of the attitude estimator. 

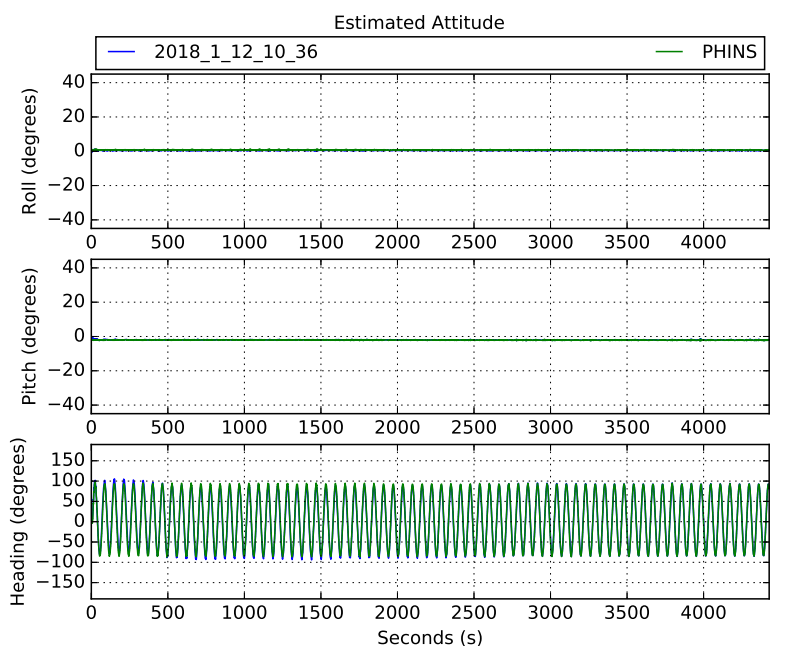

Angular Velocity Sensor Bias

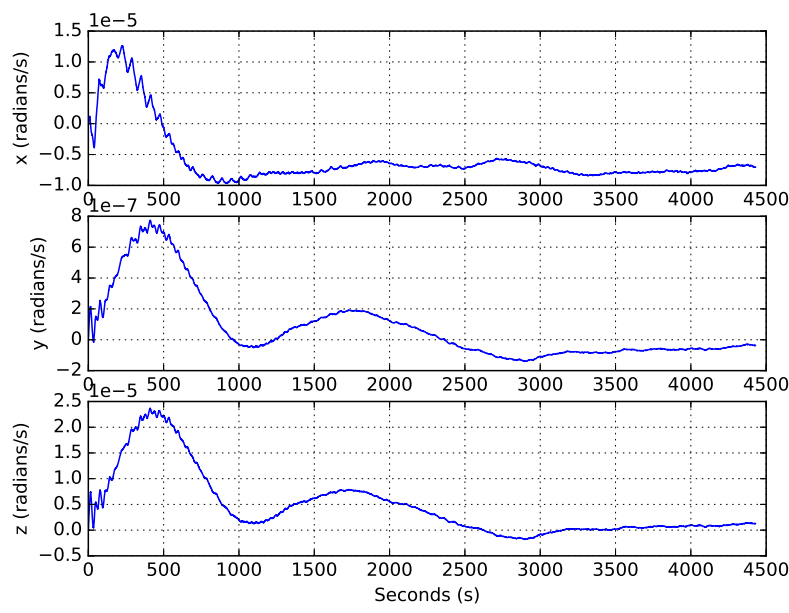

Estimated Attitude Error
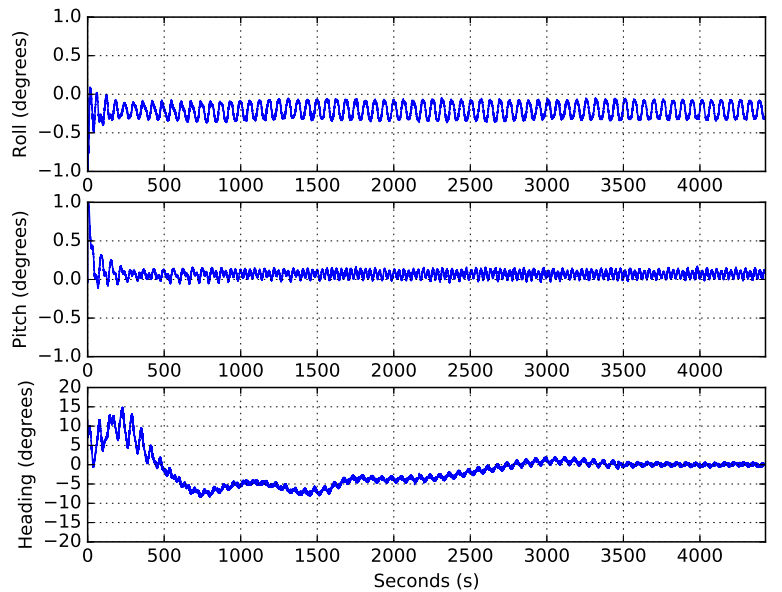

Linear Accelerometer Sensor Bias
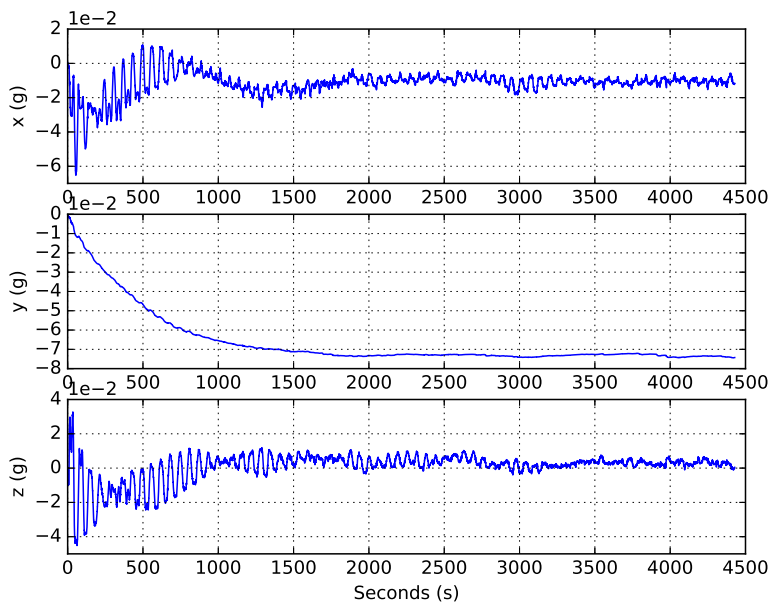

Fig. 3: Experimental results of full scale vehicle trial in test tank.

- The 2018_1_12_10_36 experiment was conducted at a latitude of $39.32^{\circ} \mathrm{N}$.

- The ROV was commanded to execute smooth sinusoidal rotations $\left(\sim 180^{\circ}\right)$ in heading while in closed-loop control.

\section{G. Experimental Results}

The attitude and sensor bias estimations and attitude errors for the vehicle trial are shown in Figure 3. The results show that during this experimental evaluation of the gyrocompass system, the attitude estimate converges to the true attitude. Roll and pitch converge to within $0.5^{\circ}$ and true-North heading to within $1^{\circ}$ of their true values.

In this preliminary result, the estimator took $\sim 50$ minutes to converge to the correct true-North heading. Long convergence time is typical of true-North gyrocompass systems. For example, the iXBlue PHINS takes $~ 25$ minutes to achieve fine alignment [13]. We are currently investigating improvements to this sensor bias and Earth-rate observer (e.g. adaptive gains) to improve its rate of convergence.

\section{CONCLUSION}

This paper reports the derivation and stability analysis of an adaptive bias and North vector observer and an attitude observer for use in true-North gyrocompass systems. Preliminary simulations and a preliminary vehicle experiment using a commercially available low-cost FOG IMU are presented.

The preliminary simulation and vehicle trial suggest, for the case of a rotating IMU, the convergence of the gyrocompass system to the true attitude without using magnetometers. The vehicle trial shows roll and pitch converge to within $0.5^{\circ}$ and true-North heading to within $1^{\circ}$ of their true values.

In future studies, the authors hope to improve the time of convergence, increase the accuracy of the gyrocompass system, extend the stability proofs to include the modified bias update law reported in Section VI-B, and do thorough vehicle trials both in the lab and in the field.

\section{ACKNOWLEDGMENTS}

We gratefully acknowledge the support of the National Science Foundation under NSF award OCE-1435818. 


\section{REFERENCES}

[1] Roberto Alonso and Malcolm D Shuster. Complete linear attitude-independent magnetometer calibration. Journal of the Astronautical Sciences, 50(4):477-490, 2002.

[2] Roberto Alonso and Malcolm D Shuster. TWOSTEP: A fast robust algorithm for attitude-independent magnetometer-bias determination. Journal of the Astronautical Sciences, 50(4):433-452, 2002.

[3] D. Clegg and M. Peterson. User Operational Evaluation System of Unmanned Underwater Vehicles for very Shallow Water Mine Countermeasures. In OCEANS 2003. Proceedings, volume 3, pages 1417-1423 Vol.3, Sept 2003.

[4] T. R. Clem, D. D. Sternlicht, J. E. Fernandez, J. L. Prater, R. Holtzapple, R. P. Gibson, J. P. Klose, and T. M. Marston. Demonstration of advanced sensors for underwater unexploded ordnance (UXO) detection. In 2012 Oceans, pages 1-4, Oct 2012.

[5] R. Costanzi, F. Fanelli, N. Monni, A. Ridolfi, and B. Allotta. An Attitude Estimation Algorithm for Mobile Robots Under Unknown Magnetic Disturbances. IEEE/ASME Transactions on Mechatronics, 21(4):19001911, Aug 2016.

[6] John L Crassidis, Kok-Lam Lai, and Richard R Harman. Real-time attitude-independent three-axis magnetometer calibration. Journal of Guidance, Control, and Dynamics, 28(1):115-120, 2005.

[7] John L Crassidis, F Landis Markley, and Yang Cheng. Survey of nonlinear attitude estimation methods. Journal of guidance, control, and dynamics, 30(1):12-28, 2007.

[8] B Gambhir. Determination of magnetometer biases using module RESIDG. Computer Sciences Corporation, Report, (3000-32700):01, 1975.

[9] Michael George and Salah Sukkarieh. Tightly coupled INS/GPS with bias estimation for UAV applications. In Proceedings of Australiasian Conference on Robotics and Automation (ACRA), 2005.

[10] Pengfei Guo, Haitao Qiu, Yunchun Yang, and Zhang Ren. The soft iron and hard iron calibration method using extended Kalman filter for attitude and heading reference system. In Position, Location and Navigation Symposium, 2008 IEEE/ION, pages 1167-1174. IEEE, 2008.

[11] Tarek Hamel and Robert Mahony. Attitude estimation on $\mathrm{SO}$ (3) based on direct inertial measurements. In Robotics and Automation, 2006. ICRA 2006. Proceedings 2006 IEEE International Conference on, pages 2170-2175. IEEE, 2006.

[12] iXblue SAS, Cedex, France. iXSEA PHINS INS datasheet.

[13] IXSEA. PHINS III User Guide. IXSEA, MU-PHINSIII006 B edition, December 2008.

[14] J. C. Kinsey and L. L. Whitcomb. Adaptive Identification on the Group of Rigid Body Rotations. In Proceedings of the 2005 IEEE International Conference on Robotics and Automation, pages 3256-3261, April 2005.

[15] James C Kinsey and Louis L Whitcomb. Preliminary field experience with the DVLNAV integrated navigation system for oceanographic submersibles. Control Engineering Practice, 12(12):1541 - 1549, 2004. Guidance and control of underwater vehicles.

[16] Manon Kok, Jeroen D Hol, Thomas B Schön, Fredrik Gustafsson, and Henk Luinge. Calibration of a magnetometer in combination with inertial sensors. In Information Fusion (FUSION), 2012 15th International Conference on, pages 787-793. IEEE, 2012.

[17] KVH Industries, Inc., Middletown, RI, USA. KVH 1775 IMU datasheet, 2015.

[18] Xiang Li and Zhi Li. A new calibration method for tri-axial field sensors in strap-down navigation systems. Measurement Science and technology, 23(10):105105, 2012.

[19] R. Mahony, T. Hamel, and J. M. Pflimlin. Nonlinear Complementary Filters on the Special Orthogonal Group. IEEE Transactions on Automatic Control, 53(5):12031218, June 2008.

[20] A. Martinelli. Vision and IMU Data Fusion: ClosedForm Solutions for Attitude, Speed, Absolute Scale, and Bias Determination. IEEE Transactions on Robotics, 28 (1):44-60, Feb 2012.

[21] N. Metni, J. M. Pflimlin, T. Hamel, and P. Soueres. Attitude and gyro bias estimation for a flying UAV. In 2005 IEEE/RSJ International Conference on Intelligent Robots and Systems, pages 1114-1120, Aug 2005.

[22] Najib Metni, Jean-Michel Pflimlin, Tarek Hamel, and Philippe Soures. Attitude and gyro bias estimation for a VTOL UAV. Control Engineering Practice, 14(12):1511 - 1520, 2006.

[23] Richard M. Murray, Zexiang Li, and S. Shankar Sastry. A Mathematical Introduction to Robotic Manipulation. CRC Press, Boca Raton, 1994.

[24] Kumpati S Narendra and Anuradha M Annaswamy. Stable adaptive systems. Prentice-Hall Inc., 1989.

[25] G. E. Packard, A. Kukulya, T. Austin, M. Dennett, R. Littlefield, G. Packard, M. Purcell, R. Stokey, and G. Skomal. Continuous autonomous tracking and imaging of white sharks and basking sharks using a REMUS100 AUV. In 2013 OCEANS - San Diego, pages 1-5, Sept 2013.

[26] F. C. Park. The Optimal Kinematic Design of Mechanisms. PhD thesis, Harvard University, Cambridge, MA USA, May 1991.

[27] J. M. Pflimlin, T. Hamel, and P. Soures. Nonlinear attitude and gyroscope's bias estimation for a VTOL UAV. International Journal of Systems Science, 38(3): 197-210, 2007.

[28] Shankar Sastry and Marc Bodson. Adaptive control: stability, convergence and robustness. Prentice-Hall Inc., 1989.

[29] G. G. Scandaroli and P. Morin. Nonlinear filter design for pose and IMU bias estimation. In 2011 IEEE 
International Conference on Robotics and Automation, pages 4524-4530, May 2011.

[30] G. G. Scandaroli, P. Morin, and G. Silveira. A nonlinear observer approach for concurrent estimation of pose, IMU bias and camera-to-IMU rotation. In 2011 IEEE/RSJ International Conference on Intelligent Robots and Systems, pages 3335-3341, Sept 2011.

[31] A. R. Spielvogel and L. L. Whitcomb. Preliminary results with a low-cost fiber-optic gyrocompass system. In OCEANS 2015 - MTS/IEEE Washington, pages 1-5, Oct 2015.

[32] A. R. Spielvogel and L. L. Whitcomb. A stable adaptive attitude estimator on $\mathrm{SO}(3)$ for true-North seeking gyrocompass systems: Theory and preliminary simulation evaluation. In 2017 IEEE International Conference on Robotics and Automation (ICRA), pages 3231-3236, May 2017.

[33] A. R. Spielvogel and L. L. Whitcomb. Adaptive estimation of measurement bias in six degree of freedom inertial measurement units: Theory and preliminary simulation evaluation. In 2017 IEEE/RSJ International Conference on Intelligent Robots and Systems (IROS), pages 5880-
5885, Sept 2017.

[34] E. Steele, T. Boyd, M. Inall, E. Dumont, and C. Griffiths. Cooling of the West Spitsbergen Current: AUV-based turbulence measurements west of Svalbard. In 2012 IEEE/OES Autonomous Underwater Vehicles (AUV), pages 1-7, Sept 2012.

[35] Giancarlo Troni and Louis L Whitcomb. Adaptive Estimation of Measurement Bias in Three-Dimensional Field Sensors with Angular Rate Sensors: Theory and Comparative Experimental Evaluation. In Robotics: Science and Systems, 2013.

[36] Woodman, Oliver J. An introduction to inertial navigation. Technical report, University of Cambridge, Cambridge, UK, 2007.

[37] T. H. Wu, E. Kaufman, and T. Lee. Globally Asymptotically Stable Attitude Observer on SO(3). In 2015 54th IEEE Conference on Decision and Control (CDC), pages 2164-2168, Dec 2015.

[38] M. Zhou, R. Bachmayer, and B. de Young. Working towards seafloor and underwater iceberg mapping with a Slocum glider. In 2014 IEEE/OES Autonomous Underwater Vehicles (AUV), pages 1-5, Oct 2014. 